\title{
Uma nova espécie e novos nomes em Piper seção Ottonia (Piperaceae) para o Sudeste do Brasil
}

\author{
Elsie Franklin Guimarães ${ }^{1,3}$ e Micheline Carvalho-Silva ${ }^{2}$
}

Recebido: 01.03.2007; aceito: 23.07.2009

ABSTRACT - (A new species and new names in Piper section Ottonia (Piperaceae) in Southeast Brazil). Trough continuous studies developed on Piperaceae of Brazil, a new species of Piper section Ottonia was found in the Rio Doce State Park. Piper rio-docense is an endemic species to the State of Minas Gerais, this is a very distinctive taxon, especially by its bullate and villous leaves. New names are provided for two species which were included in the genus Ottonia Sprengel: Piper carautensei E.F. Guim. \& M. Carvalho-Silva and Piper duartei E.F. Guim. \& M. Carvalho-Silva. Ottonia vilosa var. minensis Guimarães is a new synonym.

Key words: flora, morphology, taxonomy

RESUMO - (Uma nova espécie e novos nomes em Piper seção Ottonia (Piperaceae) para o sudeste do Brasil). Uma nova espécie de Piper L. pertencente à seção Ottonia (Spreng.) Tebbs - Piper rio-docense E.F. Guim. \& M. Carvalho-Silva encontrada no Parque Estadual do Rio Doce é descrita e ilustrada. A espécie é endêmica para o estado de Minas Gerais, muito característica, especialmente pela suas folhas buladas e providas de tricomas. Nomes novos para duas espécies que se encontravam inseridas no gênero Ottonia Sprengel são apresentados: Piper carautensei E.F. Guim. \& M. Carvalho-Silva e Piper duartei E.F. Guim. \& M. Carvalho-Silva. Ottonia vilosa var. minensis Guimarães constituiu um novo sinônimo Palavras-chave: flora, morfologia, taxonomia.

\section{Introdução}

A família Piperaceae, uma das maiores dentre as angiospermas basais, apresenta distribuição pantropical, com centro de diversidade localizado nas Américas Central e do Sul (Yuncker 1958, Jaramillo \& Manos 2001, Medeiros \& Guimarães 2007) com cerca de 2500 espécies. Engloba cinco gêneros dos quais Piper L. e Peperomia Ruiz \& Pav. são os mais diversos. O Brasil está representado por aproximadamente 500 espécies (Yuncker, 1966, 1972, 1973, 1974) distribuídas nos gêneros Piper, Peperomia e Manekia Trel. (Tebbs 1989, Jaramillo \& Manos 2001, Arias et al. 2006).

Com relação às espécies brasileiras, além da revisão da família elaborada por Yuncker (1972, 1973, 1974), vários trabalhos mais recentes de taxonomia e floras regionais foram publicados (Guimarães 1984a, 1988, 1994, Callejas 1986, Tebbs 1989, Guimarães et al. 1977, 1978, 1984b, Guimarães \& Valente 2001, Carvalho-Silva \& Cavalcanti 2002, Ruschel 2004). Tebbs $(1989,1993)$ revisou quatro seções de Piper para o Novo Mundo Macrostachys Miq., Churumayu Miq., Lepianthes Raf. e Radula Miq., nos quais considerou também Ottonia Spreng., Enkea Miq. e Callianira Miq. como seções.

Análises filogenéticas confirmaram a monofilia de grupos infragenéricos em Piper dentre eles Ottonia (Jaramillo \& Manos 2001). Espécies de Ottonia com ocorrência na floresta atlântica, são reconhecidas pelas folhas pinadas, as flores bissexuais laxamente dispostas com quatro estames e quatro carpelos, protegidas por bráctea cuculada. Nesse trabalho a autora descreveu a morfologia, distribuição geográfica, ilustrações e comenta sobre a ecologia dos grupos.

Callejas (1986), em sua tese de doutorado, revisou o gênero Ottonia onde descreveu 21 especies.

Devido às dificuldades que vem sendo encontradas quando da contextualização das Piperaceae, no âmbito da taxonomia, na medida em que há mudanças nos epítetos, cuja nomenclatura ainda não foi atualizada, propõe-se este trabalho que objetiva atualizar os novos nomes das espécies de Piper, inicialmente inseridos em Ottonia, além da descrição de um novo táxon.

1. Instituto de Pesquisas Jardim Botânico do Rio de Janeiro, Rua Pacheco Leão 915, 2240-030 Rio de Janeiro, RJ, Brasil

2. Escola Nacional de Botânica Tropical, Instituto de Pesquisas do Jardim Botânico do Rio de Janeiro

3. Autor para correspondência.eguimar@jbrj.gov.br 


\section{Material e Métodos}

O trabalho teve por base o estudo morfológico de espécimes dos herbários BHCB, CEN, MBM, RB e VIC cujas siglas seguem Holmgren et al. (1990). As medidas apresentadas na descrição da espécie nova indicam os limites mínimos e máximos encontrados. Para a observação morfológica externa foram utilizados os conceitos de Rizzini (1977) e Stearn (1998).

Foi elaborada chave analítica para as espécies estudadas que no texto segue ordem alfabética. A ilustração foi realizada em microscópio Carl Zeiss, equipado com câmara clara acoplada.

\section{Resultados e Discussão}

Tendo em vista que as espécies analisadas estão inseridas naquelas que possuem inflorescências com flores pediceladas e são semelhantes, apresenta-se chave analítica para distingui-las.

Chave analítica para as espécies estudadas

1. Folhas buladas Piper rio-docense

1. Folhas não buladas

2. Folhas peltadas, brácteas florais glabras Piper carautensei

2. Folhas não peltadas, brácteas florais providas de tricomas Piper duartei

Piper carautensei E.F. Guim. \& M. Carvalho-Silva, nom. nov.

Basiônimo: Ottonia peltata Guimarães \& Ichaso, Arq. Jard. Bot. Rio de Janeiro 20.355. 1977, non Piper peltatum L. 1753. Tipo: BRASIL. Espírito SANTO: Domingo Martins, Campinho, rod. do Chapéu, 15-IX1975, fr., J.P. Carauta 1808 (holótipo RB)

Piper carautensei é endêmica do Espírito Santo e ocorre em altitudes que variam de $630-700 \mathrm{~m}$ s.m. O epíteto foi dado em homenagem ao coletor do Holótipo, J.P. Carauta, botânico estudioso das Moraceae.É espécie muito semelhante a Piper duartei E.F. Guim \& M. Carvalho-Silva, principalmente pela pilosidade invulgar nestas espécies da seção. As folhas peltadas com tricomas mais esparsos, pedicelos mais longos e brácteas florais glabras são os caracteres que a distinguem de $P$. duartei. O nome Piper guimaraesianum foi utilizado por Callejas (1986) em sua tese de doutorado e não publicado.
Material examinado: BRASIL. Espírito SAnTo: Alfredo Chaves, São Bento de Urânia, 8-X-1994, G. Hatschbach \& J.M. Silva 61164 (CEN, MBM); Santa Maria de Jetiba, Belém, terreno de Paulo Seick (área 1), 19-IX-2000, L. Kollmann 5768 (RB); Domingos Martins, 18-I-1975, A.L. Peixoto 438 (RB); Santa Tereza, Estação Biológica de Santa Lúcia, 19-IX1988, B. Fernandes 2540 (RB); idem, na área da Reserva Biológica Santa Lúcia, trilha para o túmulo de Augusto Ruschi, 13-IX-2006, R. Marquete et al. 3887 (RB); idem, Estrada para Santa Leopoldina, 13IX-2006, R. Marquete et al. 3901 (RB).

Piper duartei E.F. Guim. \& M. Carvalho-Silva, nom. nov.

Basiônimo: Ottonia villosa Yuncker, Bol. Inst. Bot. São Paulo. 3: 139. 1966, non Piper villosum C. DC. 1866. Tipo: BRASIL. Espírito SANTo: Santa Tereza, 25-XI-1953, fl., fr., A.P. Duarte 4012 (holótipo $\mathrm{RB})$.

= Ottonia vilosa var. minensis Guimarães, Napaea 5: 13. 1988. Tipo: BRASIL. MinAS Gerais: Caratinaga, Est. Biol. Caratinaga, 21-IX-1984, P.M. Andrade \& M.A. Lopes 334 (holótipo RB), syn. nov.

Piper duartei ocorre nos estados do Espírito Santo e Minas Gerais. O epíteto foi dado em homenagem ao coletor do holótipo A.P. Duarte, botânico do Jardim Botânico do Rio de Janeiro. A espécie é morfologicamente próxima a Piper carautensei da qual difere por apresentar folhas não peltadas com pilosidade mais acentuada, pedicelos mais curtos e bractéolas providas de tricomas. Ottonia villosa var. minensis E.F. Guim. foi sinonimizada com base na análise do material-tipo da variedade, entre outros exemplares, que demonstraram que a relação pedicelo = bráctea floral e o comprimento das espigas não se apresentaram constantes nos materiais examinados, o que a aproximou morfologicamente de Piper duartei. O nome Piper villoso-bracteatum foi utilizado por Callejas (1986) em sua tese de doutorado, mas não publicado.

Material examinado: BRASIL: EspíRITo SANTo. Itarana, caminho para Alto Limoeiro, 11-VI-2005, M.A. Jaramillo et al. 956 (RB); Santa Tereza, 27I-1999, L. Kollmann 1696 (RB); idem, Reserva Biológica Augusto Ruschi, estrada para GoiapabaAçu, 10-VI-2005, M.A. Jaramillo et al. 942 (RB); idem, São Lourenço, 2-XII-1998, L. Kollmann 1153 (RB); idem, mata fria, terreno de Clerio Lopes, 10-VI2005, M.A. Jaramillo et al. 928 (RB). Minas GeraIs: 
Caratinga, Estação Biológica de Caratinga, Fazenda Montes Claros, trilha M1, 1943'53"S, 41 499'2'W, 6-IX-1998, J.A. Lombardi et al. 2367 (BHCB, CEN, RB); idem, 22-II-1984, M.A. Lopes 180 (RB); idem, 21-IX-1984, P.M. Andrade 334 (RB); idem, 21-IV1984, M.A. Lopes 255 (RB); idem, 21-IX-1984, P.M. Andrade 338 (RB); Serra da Moeda, km 2 da estrada da Moeda, 20¹8'S, 4358'W, 15-XI-1987, V.C.F.M. s.n (RB331775).

Piper rio-docense E.F. Guim. \& M. Carvalho-Silva. sp. nov. Tipo: BRASIL. Minas Gerais: Parque Estadual do Rio Doce, trilha do Vinhático, 11-XII1999, fl., G.E. Valente et al. 430 (holótipo VIC, isótipo $\mathrm{RB})$.

Frutex 0,35-1 m altus, ramis villosis, $2 \mathrm{~mm}$ diam . Folia supra bullata, subtus lacunosa, membranacea vel papiracea, elliptica vel oblongo-lanceolata, lamina equilatera, discolore, basi cordata vel lobata; lobis supra petiolos, apice acuto vel attenuatoacuminato, adaxialiter glabra, abaxialiter villosa, nervis 9-13, supra impressos, subter prominentibus; petiolus 3,5-7 mm, villosus; prophyllum caducum, 0,8-1 cm longum, acuminatum, supra villosum, subter glabrum. Inflorescentia erecta, alba; pedunculus 3-5 mm longus, villosus; rachis 3,5-7,3 cm longa, striata, papillosa, glabra, obscure glandulosa; pedicellus 0,8-2 mm longus; flores laxi, antheris 0,3 mm latis, ovário 0,4-0,5 mm longo, stigmatibus 4, sessilibus, papillosis. Fructus 1,5 mm longi, 0,5-0,7 mm lati, elliptici, castanei, papillosi, 4-angulosi.

Subarbusto 0,35-1 m, ramos vilosos, ca. $2 \mathrm{~mm}$ diâm. Folhas buladas na face adaxial, lacunosas na abaxial, membranáceas ou papiráceas, elípticas ou oblongo-lanceolada; lâmina equilatera discolor (8-)10,5-14 cm compr. 3-4,3 cm larg., base cordada ou lobada, lobos sobre os pecíolos, ápice agudo ou atenuado-acuminado, face adaxial glabra, face abaxial vilosa, nervuras 9-13, impressas na face adaxial, proeminentes na abaxial; pecíolos 3,5-7 mm compr., vilosos. Profilo caduco, 0,8-1 cm compr., acuminado, viloso na face adaxial e glabro na abaxial. Inflorescências eretas, alvas; pedúnculo 3-5 mm, viloso; raque 3,5-7,3 cm compr., estriada, papilosa, glabra, obscuramente glandulosa; bráctea floral 0,3$0,5 \mathrm{~mm}$ larg., sacado-galeada, glandulosa, pedicelos 0,8-2 mm compr. Flores laxas, anteras $0,3 \mathrm{~mm}$ larg., ovário ca. 0,5 mm compr, estigmas 4 , sésseis, papilosos. Frutos castanhos, elipsóides, 4-angulosos, papilosos, 1,5 mm compr., 0,5-0,7 mm diâmetro.
Piper rio-docence E.F. Guim. \& M. CarvalhoSilva é uma espécie endêmica de Minas Gerais do Parque Estadual do Rio Doce. O epíteto específico refere-se à localidade-tipo da planta. Caracteriza-se principalmente pelas folhas buladas, que chamam a atenção por ser o primeiro registro na seção Ottonia. A pilosidade é um caráter diferencial para as espécies deste grupo, que em sua maioria são glabras, assim a espécie assemelha-se a Piper carautensei e Piper duartei, também profusamente pilosas.

Quando da análise dos exemplares do RB verificou-se que havia nome na exsicata que ainda não havia sido publicado o que conduziu as autoras a publicar esta espécie.

Material adicional examinado: BRASIL. MinAS GERAIS: Marliéria, Parque Estadual do Rio Doce, trilha para a Lagoa Preta, 24-VIII-1998, fl,, M.G. Bovini \& Marquinhos 1516 (VIC); Forest Reserve of Rio Doce, forests of Lagoa Central, 190-200 m, 30-VIII-1973, fr., D. Sucre et al. 10133 (RB).

$\mathrm{O}$ acesso à coleção do herbário da Universidade de Viçosa (VIC) para o estudo das Piperaceae de Minas Gerais permitiu a identificação desta nova espécies, Piper rio-docense E.F. Guim \& M. CarvalhoSilva que apresenta como principal característica as flores e os frutos pedicelados, próprios da seção Ottonia (Spreng.) Tebbs. Trata-se de uma espécie pouco coletada representada apenas nos herbários RB e VIC, oriunda de uma única localidade, o Parque Estadual do Rio Doce, Minas Gerais.

Neste trabalho seguiu-se o conceito de Tebbs (1989) que considerou o gênero Ottonia Sprengel como seção Ottonia (Spreng.) Tebbs de Piper L. Por outro lado Callejas (1986) em sua tese de doutorado (dados não publicados) também considerou Ottonia como um táxon de Piper.

Para este grupo, que apresenta inflorescência com flores pediceladas, as espécies que foram estudadas revelaram caracteres bastante interessantes e de valor taxonômico, como o observado em Piper riodocense que dispõe de folhas buladas na face adaxial e lacunosas na abaxial , além da pilosidade que também está expressa em $P$.duartei, caracteres diferencias para os táxons analisados que, geralmente, são glabros, exceto pela presença de pilosidade na nervura mediana da face abaxial como em Piper anisum (Spreng.) Angely.

Ressalta-se ainda que a morfologia foliar no que se refere às folhas peltadas, foi um caráter peculiar para as espécies da seção. 


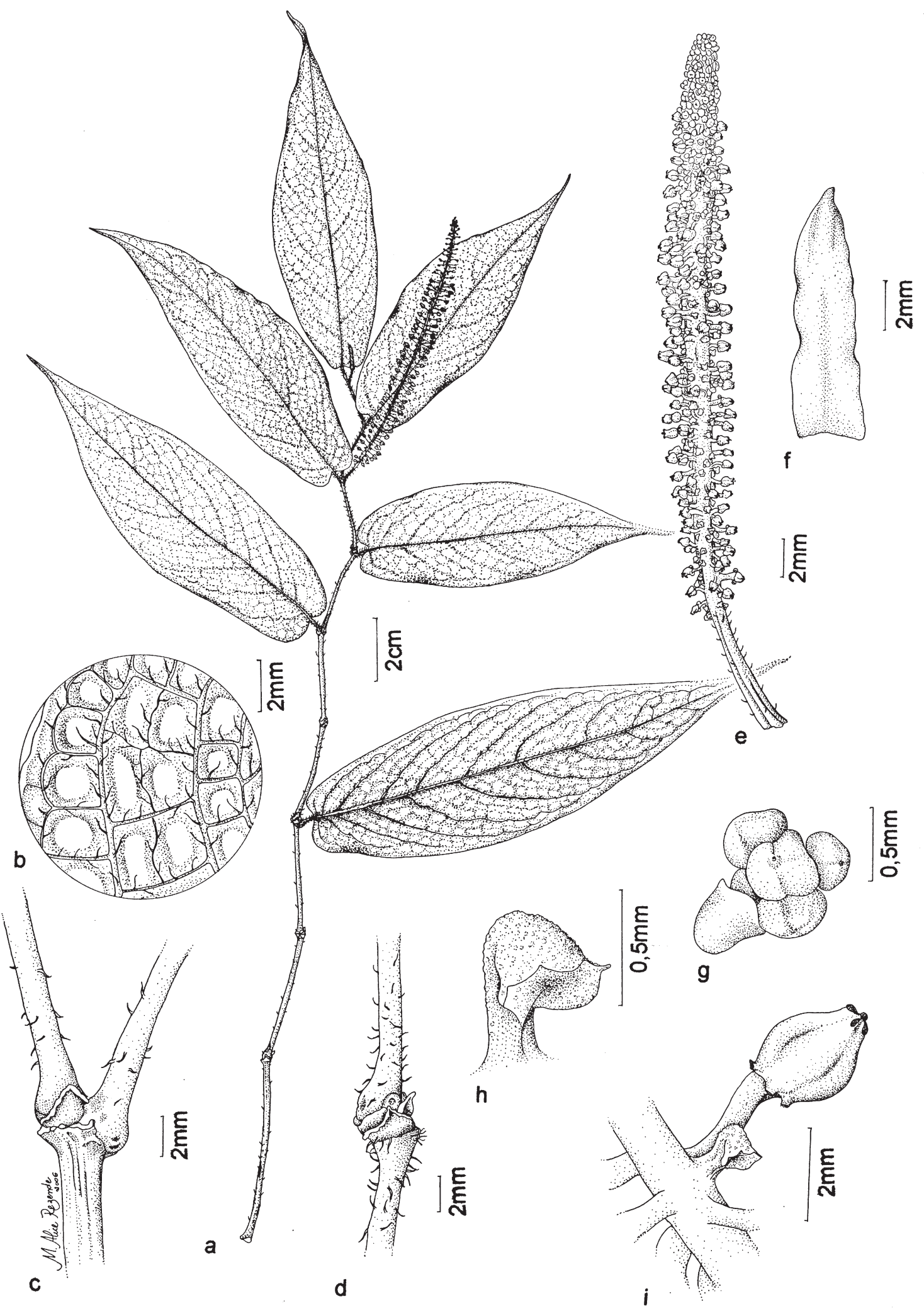

Figura 1. Piper rio-docense E.F. Guim. \& M. Carvalho-Silva. a. Hábito. b. Detalhe da lâmina foliar face abaxial. c. Detalhe dos ramos. d. Detalhe do pedúnculo. e. Inflorescência. f. Profilo. g. Detalhe da flor. h. Detalhe da bráctea floral. i. Fruto e bráctea floral.

Figure 1. Piper rio-docense E.F. Guim. \& M. Carvalho-Silva. a. Habit. b. Detail of the abaxial leaf surface. c. Branch detail. d. Peduncle. e. Inflorescence. f. Prophyllum. g. Flower h. Floral bract. i. Fruit and floral bract. 
O trabalho de Tebbs (1989) permitiu também que novos nomes da seção Ottonia fossem estabelecidos: Piper carautensei E.F. Guim. \& M. Carvalho-Silva para Ottonia peltata E.F. Guim. \& Ichaso, e Piper duartei E.F. Guim \& M. Carvalho-Silva, para Ottonia villosa Yunck.

O táxon Ottonia villosa var. minensis E.F. Guim., foi sinonimizado sob Piper duartei E.F. Guim \& M. Carvalho-Silva, em decorrência da superposição das similaridades encontradas nos diferentes materiais examinados.

\section{Agradecimentos}

Ao conselho Nacional de Desenvolvimento Científico e Tecnológico (CNPq) e à Coordenação de Aperfeiçoamento de Pessoal de Nível Superior (CAPES), pelas bolsas concedidas as autoras; à Petrobrás (processo 610.4.025.02.3 )/Programa Mata Atlântica - JBRJ; aos curadores de herbário pelos materiais remetidos e ao Dr. Jorge Fontella Pereira pela correção da diagnose latina.

\section{Literatura citada}

Arias, T., Posada, R.C. \& Bornstein, A. 2006. New combinations in Manekia, an earlier name for Sarcorhachis (Piperaceae). Novon 16: 205-208.

Callejas, R. 1986. Taxonomic revision of Piper subgenus Ottonia (Piperaceae) South America. PhD Thesis, City University of New York, New York.

Carvalho-Silva, M.\& Cavalcanti,T.B.2002. Piperaceae. In: T.B. Cavalcanti \& A.E. Ramos (orgs.). Flora do Distrito Federal, v.2. Embrapa Recursos Genéticos e Biotecnologia, Brasília, pp. 1-184

Guimarães, E.F., Ichaso, C.L.F. \& Costa, C.G. 1977. Ottonia peltata (Piperaceae) uma nova espécie do Estado do Espírito Santo. Archivos Jardim Botânico do Rio de Janeiro 20: 35-40.

Guimarães, E.F., Ichaso, C.L.F. \& Costa, C.G. 1978. 1 Ottonia, 2 Sarcorhachis 3 Potomorphe. In: R. Reitz \& R.M. Klein (eds.). Flora Ilustrada Catarinense. Herbário Barbosa Rodrigues, Itajaí, pp. 1-26.

Guimarães, E.F. 1988. Uma nova variedade de Ottonia villosa (Notas em Piperaceae III). Napaea 5: 13-15.

Guimarães, E.F. 1984a. Notas em Piperaceae II. Considerações sobre o gênero Ottonia Sprengel no Brasil. Boletim Museu Botânico Kuhlmann 7: 61-85.
Guimarães, E.F., Ichaso, C.L.F. \& Costa, C.G. 1984b. Peperomia In: R. Reitz \& R.M. Klein (eds.). Flora Ilustrada Catarinense. Herbário Barbosa Rodrigues, Itajaí, pp. 1-112.

Guimarães, E.F. 1994. Piperaceae In: M.P.M. Lima \& R.R. Guedes-Bruni (orgs.). Reserva Ecológica de Macaé de Cima, Nova Friburgo, RJ. Aspectos florísticos das espécies vasculares, v. 1. Jardim Botânico do Rio de Janeiro, Rio de Janeiro, pp. 327-348.

Guimarães, E.F. \& Valente M.C. 2001. Piper In: R. Reitz \& R.M. Klein (eds.). Flora Ilustrada Catarinense. Herbário Barbosa Rodrigues, Itajaí.

Holmgreen, P.K., Holmgren, N.H. \& Barnett, L.C. 1990. Index herbariorum. Part.1: The herbaria of the world, 8 ed. New York Botanical Garden, New York.

Jaramillo, M.A. \& Manos, P.S. 2001. Phylogeny and patterns of floral diversity in the genus Piper (Piperaceae). American Journal of Botany 88: 706-716.

Medeiros, E.S. \& Gimarães, E.F. 2007. Piperaceae do Parque Estadual do Ibitipoca, Minas Gerais, Brasil. Boletim de Botânica da Universidade de São Paulo 25: 227-252.

Rizzini, C.T. 1977. Sistematização terminológica da folha. Rodriguésia 29: 103-126.

Ruschel, D. 2004. O gênero Piper (Piperaceae) no Rio Grande do Sul. Dissertação de Mestrado, Universidade Federal do Rio Grande do Sul, Porto Alegre.

Stearn, W.T. 1998. Botanical Latin. 4 ed. Timber Press, Portland.

Tebbs, M.C. 1989. Revision of Piper (Piperaceae) in the New World 1. Review of characters and taxonomy of Piper section Macrostachys. Bulletin of the Britsh Museum, Botany 19: 117-158.

Tebbs, M.C. 1993. Revision of Piper (Piperacea) in the New World 3. Taxonomy of Piper sections Lepianthes and Radula. Bulletin of the Natural History Museum, Botany series 23: 1-50

Yuncker, T.G. 1958. The Piperaceae - family profile. Brittonia 10: 1-7.

Yuncker, T.G. 1966. New species of Piperaceae from Brazil. Boletim do Instituto de Botânica 3: 1-370.

Yuncker, T.G. 1972. The Piperaceae of Brazil I: Piper Group I, II, III, IV. Hoehnea 2: 19-366.

Yuncker, T.G. 1973. The Piperaceae of Brazil II: Piper: Group V; Ottonia; Pothomorphe; Sarcorhachis. Hoehnea $3: 29-284$.

Yuncker, T.G. 1974 The Piperaceae of Brazil III: Peperomia; taxa of uncertain status. Hoehnea 4: 71-413. 
